\title{
The analytic structure of heavy quark propagators
}

\author{
C. J. Burden \\ Department of Theoretical Physics, Research School of Physical Sciences and Engineering, \\ Australian National University, Canberra, ACT 0200, Australia
}

\begin{abstract}
The renormalised quark Dyson-Schwinger equation is studied in the limit of the renormalised current heavy quark mass $m_{R} \rightarrow \infty$. We are particularly interested in the analytic pole structure of the heavy quark propagator in the complex momentum plane. Approximations in which the quark-gluon vertex is modelled by either the bare vertex or the Ball-Chiu Ansatz, and the Landau gauge gluon propagator takes either a gaussian form or a gaussian form with an ultraviolet asymptotic tail are used.
\end{abstract}

PACS NUMBERS: 12.38.Aw, 12.38.Lg, 12.39.Hg 


\section{INTRODUCTION}

The solution of approximate Dyson-Schwinger equations (DSE) has proved to be an effective means for modelling quark propagators in hadronic physics [1]. Recent calculations within the genre of models which we shall refer to as the DSE technique include those of the light hadron spectrum [2,3] and of electromagnetic form factors of the pion and kaon [4]. Although no rigorous proof exists, it is the philosophy of the DSE technique that one possible signal of confinement in QCD should be the absence of timelike poles in the quark propagator [5]. It has furthermore been conjectured that the propagator $S(p)$ could be an entire function in the complex $p^{2}$ plane [6,7]. Such a scenario would, for instance, avoid certain unpleasant consequences which can result when modelling mesons via the Bethe-Salpeter equation which samples the quark propagator over a region of the complex plane.

Determining the analytic structure of fermion propagators in QCD [8,9] or other confining theories [10,11] by the direct solution of model DSEs is not easy. It appears that the pole or branch cut structure obtained in any particular model is heavily dependent on the approximations employed. In general, two aspects of the quark DSE must be approximated: the quark-gluon vertex, and the gluon propagator. In this paper, we shall look at both these aspects within the heavy quark sector. In existing numerical studies in the light quark sector which produce propagators with conjugate singularities [8.9], the quark gluon vertex has usually been approximated by the bare vertex (the so called rainbow approximation). In ref. [7], however, it was shown that an entire function propagator can be obtained if the vertex function is modelled by a more sophisticated form respecting the Ward-Takahashi identity. This suggests that it is worthwhile exploring the importance of accurately modelling the quark-gluon vertex, as well as the gluon propagator, when studying the analytic structure of the quark propagator.

In a recent development, the DSE technique has been extended to the realm of heavy quarks [12,13] in a way inspired by heavy quark effective theory (HQET) [14]. The purpose of this exercise was twofold. Firstly, if one acknowledges the success of the DSE technique in the light quark sector, it is clear that the dynamics of confined particles is driven by non-perturbative dynamical self dressing. In HQET, non-perturbative self dressing and the detailed analytic structure of the heavy quark propagator are largely ignored. It is important to know whether this is justified, or whether the successes of HQET are purely fortuitous. Secondly, one has the hope that an accurate determination of the heavy quark propagator will eventually prove useful for building phenomenological models of heavy quark hadrons.

In ref. 12 a preliminary attempt is made to calculate the spectrum of heavy quarklight antiquark mesons by using the combination of rainbow DSE and ladder Bethe-Salpeter equation (BSE). It is found that, within the limitations of the model, the pole structure of the heavy quark propagator prevents solution of the meson BSE. This is clearly a shortcoming of the approximations involved. In ref. [13 the heavy quark DSE is examined from the point of view of the gauge technique. This is essentially an improvement on the rainbow approximation to the quark-gluon vertex which is designed to respect the Ward-Takahashi identity. An alternative approach, and one which we follow in this paper, is to replace the bare vertex Ansatz with the Ball-Chiu vertex Ansatz [15]. We shall see that in the heavy fermion limit the gauge technique and the Ball-Chiu vertex are equivalent. 
Regarding the gluon propagator, our treatment differs from ref. [13] in that it is principally numerical, enabling us to concentrate on a more realistic class of model gluon propagators. Specifically we study the simple gaussian Ansatz employed in ref. [12], designed to model the infrared enhanced behaviour of the gluon propagator, and a model proposed by Frank and Roberts (FR) [16] which includes both an enhanced infrared behaviour and the known asymptotic ultraviolet behaviour. In order to deal with the FR propagator, it has been necessary to formulate a properly renormalised version of the heavy quark DSE of ref. [12].

In summary, our main finding is as follows: Improving the vertex Ansatz does little to improve the analytic structure of the quark propagator. However, improving the Ansatz employed for the gluon propagator, particularly by including a realistic asymptotic ultraviolet tail, moves poles in the heavy quark propagator to a less intrusive part of the complex momentum plane. This portends well for future application of the heavy quark DSE technique.

The layout of the paper is as follows. In Section II we summarise the renormalised quark DSE and the approximations we shall be employing for the quark-gluon vertex and gluon propagator. In Section III we summarise the heavy quark formalism and derive a renormalised DSE for the heavy quark propagator. Numerical solutions to these equations are discussed in Section IV. Conclusions are drawn and suggestions for the direction of future work are given in Section V.

\section{THE QUARK DYSON-SCHWINGER EQUATION}

Our starting point is the renormalised quark DSE [1]

$$
\Sigma^{\prime}(p, \Lambda)=Z_{1}\left(\mu^{2}, \Lambda^{2}\right) \frac{4 g^{2}}{3} \int^{\Lambda} \frac{d^{4} q}{(2 \pi)^{4}} D_{\mu \nu}(p-q) \gamma_{\mu} S(q) \Gamma_{\nu}(q, p)
$$

where we have used a Euclidean metric in which timelike vectors satisfy $p^{2}=-p_{\text {Minkowski }}^{2}<0$, and for which $\left\{\gamma_{\mu}, \gamma_{\nu}\right\}=2 \delta_{\mu \nu}$. Our aim is to solve the DSE for the renormalised quark propagator $S(p, \mu)$, which we write in the form

$$
\begin{aligned}
S(p, \mu) & =\frac{1}{i \gamma \cdot p A\left(p^{2}, \mu^{2}\right)+B\left(p^{2}, \mu^{2}\right)} \\
& =\frac{1}{Z_{2}\left(\mu^{2}, \Lambda^{2}\right)\left[i \gamma \cdot p+m_{0}(\Lambda)\right]+\Sigma^{\prime}(p, \Lambda)} .
\end{aligned}
$$

The unrenormalised self energy is written

$$
\Sigma^{\prime}(p, \Lambda)=i \gamma \cdot p\left[A^{\prime}\left(p^{2}, \Lambda^{2}\right)-1\right]+B^{\prime}\left(p^{2}, \Lambda^{2}\right) .
$$

If the renormalisation scale is set such that

$$
\left.S(p)\right|_{p^{2}=\mu^{2}}=\frac{1}{i \gamma \cdot p+m_{R}\left(\mu^{2}\right)},
$$

it follows from Eqs. (2.2) and (2.3) that renormalised and bare quantities are related by 


$$
\begin{gathered}
Z_{2}\left(\mu^{2}, \Lambda^{2}\right)=2-A^{\prime}\left(\mu^{2}, \Lambda^{2}\right), \\
A\left(p^{2}, \mu^{2}\right)=1+A^{\prime}\left(p^{2}, \Lambda^{2}\right)-A^{\prime}\left(\mu^{2}, \Lambda^{2}\right), \\
B\left(p^{2}, \mu^{2}\right)=m_{R}\left(\mu^{2}\right)+B^{\prime}\left(p^{2}, \Lambda^{2}\right)-B^{\prime}\left(\mu^{2}, \Lambda^{2}\right) .
\end{gathered}
$$

The set of equations (2.1) and (2.5) to (2.7) together with the 'abelian approximation' $Z_{1}=Z_{2}$ can be solved numerically for the propagator functions $A$ and $B$ once the renormalised quark-gluon vertex function $\Gamma_{\mu}$, the renormalised gluon propagator $D_{\mu \nu}$, the renormalisation point $\left(\mu, m_{R}(\mu)\right)$ and cutoff $\Lambda$ are specified. The precise forms of the quark-gluon vertex and gluon propagator are unknown and must be modelled by appropriate Ansätze. We next summarise the Ansätze employed in this paper.

\section{A. Quark-gluon vertex}

The most general form of the quark-gluon vertex consistent with Lorentz and CPT invariance, satisfying the Ward identity $i \Gamma_{\mu}(p, p)=\partial_{\mu}^{p} S^{-1}(p)$ and Ward Takahashi identity円 $i(p-q)_{\mu} \Gamma_{\mu}(p, q)=S^{-1}(p)-S^{-1}(q)$, and free of kinematic singularities has been given by Ball and Chiu [15]. It takes the form

$$
\Gamma_{\mu}(p, q)=\Gamma_{\mu}^{\mathrm{BC}}(p, q)+\Gamma_{\mu}^{\mathrm{T}}(p, q)
$$

where

$$
\begin{aligned}
\Gamma_{\mu}^{\mathrm{BC}}(p, q) & =\frac{1}{2}\left[A\left(p^{2}\right)+A\left(q^{2}\right)\right] \gamma_{\mu} \\
& +\frac{(p+q)_{\mu}}{\left(p^{2}-q^{2}\right)}\left\{\left[A\left(p^{2}\right)-A\left(q^{2}\right)\right] \frac{\gamma \cdot p+\gamma \cdot q}{2}-i\left[B\left(p^{2}\right)-B\left(q^{2}\right)\right]\right\},
\end{aligned}
$$

and $\Gamma_{\mu}^{\mathrm{T}}(p, q)$ is an otherwise unconstrained piece satisfying the conditions $(p-q)_{\mu} \Gamma_{\mu}^{\mathrm{T}}(p, q)=0$ and $\Gamma_{\mu}^{\mathrm{T}}(p, p)=0$.

We mention two well studied vertex Ansätze falling within this class. The first of these, introduced by Curtis and Pennington [17] to ensure multiplicative renormalisability in quantum electrodynamics, is defined by setting the transverse piece $\Gamma_{\mu}^{\mathrm{T}}$ equal to

$$
\Gamma_{\mu}^{\mathrm{T}_{\mathrm{CP}}}(p, q)=\frac{A\left(p^{2}\right)-A\left(q^{2}\right)}{2 d(p, q)}\left[\gamma_{\mu}\left(p^{2}-q^{2}\right)-(p+q)_{\mu}(\gamma \cdot p-\gamma \cdot q)\right]
$$

with

\footnotetext{
${ }^{1}$ Strictly speaking, it is the Slavnov-Taylor identities, which include ghost contributions, and not the Ward-Takahashi identities which are relevant to QCD. By using the Ball-Chiu vertex we are effectively ignoring the ghost self energy and ghost-quark scattering kernel. This is a commonly used approximation [1].
} 


$$
d(p, q)=\frac{\left(p^{2}-q^{2}\right)^{2}+\left[M^{2}\left(p^{2}\right)+M^{2}\left(q^{2}\right)^{2}\right]^{2}}{p^{2}+q^{2}}
$$

where $M=B / A$.

The second of these, proposed by Haeri [18], takes the form

$$
\begin{aligned}
\Gamma_{\mu}^{\mathrm{H}}(p, q)= & \frac{p^{2} A\left(p^{2}\right)-q^{2} A\left(q^{2}\right)}{p^{2}-q^{2}} \gamma_{\mu}+\frac{A\left(p^{2}\right)-A\left(q^{2}\right)}{p^{2}-q^{2}} \gamma \cdot p \gamma_{\mu} \gamma \cdot q \\
& -i \frac{B\left(p^{2}\right)-B\left(q^{2}\right)}{p^{2}-q^{2}}\left(\gamma \cdot p \gamma_{\mu}+\gamma_{\mu} \gamma \cdot q\right) .
\end{aligned}
$$

It satisfies the above criteria and therefore must be of the form Eq. (2.8). Munczek [19] has shown that the Haeri vertex is identical to the spectral representation of the vertex used in the gauge technique, and this has in turn been employed in ref. [13].

\section{B. Gluon propagator}

In a general covariant gauge, the gluon propagator takes the form

$$
g^{2} D_{\mu \nu}(k)=\left(\delta_{\mu \nu}-\frac{k_{\mu} k_{\nu}}{k^{2}}\right) \Delta\left(k^{2}\right)+g^{2} \xi \frac{k_{\mu} k_{\nu}}{k^{4}},
$$

where $\xi$ is the gauge fixing parameter. Perhaps the simplest Ansatz which has proved useful for modelling QCD is the 'infrared dominant' model [6, []

$$
\Delta_{\mathrm{IR}}\left(k^{2}\right)=\frac{3}{16}(2 \pi)^{4} \mu^{2} \delta^{4}(k)
$$

In applications to hadronic physics, $\mu$ is usually taken to be of the order of $1 \mathrm{GeV}$ [20], which is the typical scale of QCD. An obvious disadvantage of this model is that it neglects completely any intermediate or ultraviolet behaviour. One can go some way towards incorporating some intermediate energy structure by using the computationally convenient gaussian model

$$
\Delta_{\mathrm{G}}\left(k^{2}\right)=\frac{3}{16}(2 \pi)^{4} \frac{\mu^{2}}{\alpha^{2} \pi^{2}} e^{-k^{2} / \alpha} .
$$

We note that the infrared dominant gluon model $\Delta_{\mathrm{IR}}$, together with the minimal Ball-Chiu vertex $\Gamma_{\mu \nu}(p, q)=\Gamma_{\mu \nu}^{\mathrm{BC}}(p, q)$ defines precisely the light quark model considered in ref. [7], whereas the gaussian model $\Delta_{\mathrm{G}}$ has been used in our earlier rainbow approximation studies of heavy quarks [12]. Below we shall explore the effect on the analytic structure of the heavy quark propagator of combining either $\Delta_{\mathrm{IR}}$ or $\Delta_{\mathrm{G}}$ with the Ball-Chiu vertex.

A more realistic model gluon propagator which goes some way toward modelling the asymptotically free ultraviolet behaviour of QCD (neglecting logarithmic corrections) has been proposed by Frank and Roberts [16]. It takes the form

$$
\Delta_{\mathrm{FR}}\left(k^{2}\right)=4 \pi^{2} d\left[4 \pi^{2} m_{t}^{2} \delta^{4}(k)+\frac{1-e^{k^{2} /\left(4 m_{t}^{2}\right)}}{k^{2}}\right],
$$


where $d=12 /\left(33-2 N_{f}\right), N_{f}=3$ is the number of light quark flavours, and $m_{t} \approx 0.69 \mathrm{GeV}$ is a parameter fitted to a range of calculated pion observables. In our numerical calculations, we find it more convenient to consider a gaussian smeared version of the FR propagator given by

$$
\Delta_{\mathrm{GFR}}\left(k^{2}\right)=(2 \pi)^{4} \frac{m_{t}^{2} d}{\alpha^{2} \pi^{2}} e^{-k^{2} / \alpha}+4 \pi^{2} d \frac{1-e^{k^{2} /\left(4 m_{t}^{2}\right)}}{k^{2}} .
$$

This will enable comparisons to be made with the pure gaussian model $\Delta_{G}$.

In the next section we consider the heavy quark limit of the quark Dyson-Schwinger equation. Our earlier analysis of this limit [12] employed the heavily damped gluon propagator $\Delta_{\mathrm{G}}$, and so was free from ultraviolet divergences. In order to deal with a more realistic model, such as Eq. (2.17), it is necessary to develop a properly renormalised version of the formalism.

\section{THE HEAVY QUARK LIMIT}

\section{A. Heavy quark propagator}

In the absolute limit of heavy renormalised quark masses, $m_{R}\left(\mu^{2}\right) \rightarrow \infty$, the dressed quark propagator Eq. (2.2) is dominated by the bare form $S_{\text {bare }}^{-1}=i \gamma \cdot p+m_{R}$. However it is important to isolate from the full inverse propagator finite order self energy corrections to the bare inverse propagator which drive confining and remnant chiral symmetry breaking effects. To this end we write the momentum variables occurring in the DSE Eq. (2.1) as

$$
p_{\mu}=i m_{R}\left(\mu^{2}\right) v_{\mu}+k_{\mu}, \quad q_{\mu}=i m_{R}\left(\mu^{2}\right) v_{\mu}+k_{\mu}^{\prime},
$$

where, for convenience, we take $v=(\mathbf{0}, 1)$, so $k \cdot v=k_{4}$. We then write the renormalised quark propagator functions as

$$
\begin{gathered}
A\left(p^{2}, \mu^{2}\right)=1+\frac{\Sigma_{A}(K, \kappa)}{m_{R}\left(\mu^{2}\right)}, \\
B\left(p^{2}, \mu^{2}\right)=m_{R}\left(\mu^{2}\right)+\Sigma_{B}(K, \kappa),
\end{gathered}
$$

where we have defined the independent momentum variable

$$
K=\frac{p^{2}+m_{R}^{2}}{2 i m_{R}}=k_{4}+\frac{k^{2}}{2 i m_{R}},
$$

and renormalisation point

$$
\kappa=\frac{\mu^{2}+m_{R}^{2}}{2 i m_{R}}
$$

in the complex $K$ plane. The change of dependent variable $p^{2} \rightarrow K$ induced by the transformation Eq. (3.21) is illustrated in Fig. 1. In general, when working to zeroth order in $1 / m_{R}$, 
one can use the approximation $K=k_{4}$ (an approximation which was used in ref. [12]). An exception to this rule, relevant to models such as Eq. (2.17) for which the gluon propagator is not heavily ultraviolet damped, is in the denominator of the heavy quark propagator in the integrand in the quark DSE. This point will become clearer at the end of Section IIIB.

From Eqs. (2.2), (3.19) and (3.20) we have

$$
S(p, \mu)=\frac{1+\gamma_{4}}{2} \frac{1}{i K+\Sigma(K, \kappa)}+O\left(\frac{1}{m_{R}}\right)
$$

where we have defined the heavy quark self energy

$$
\Sigma(K, \kappa)=\Sigma_{B}(K, \kappa)-\Sigma_{A}(K, \kappa) .
$$

We find in general that the DSE leads to a single integral equation for the complex valued function $\Sigma(K, \kappa)$. The form given by Eq. (3.23) represents the heavy quark propagator in the dominant region near the the bare propagator mass pole $p^{2}=-m_{R}^{2}$. Obtaining an integral equation for $\Sigma(K)$ involves the change of integration $\int d^{4} q \rightarrow \int d^{4} k^{\prime}=\int_{-\infty}^{\infty} d k_{4}^{\prime} \int_{0}^{\infty} d|\mathbf{k}||\mathbf{k}|^{2}$ induced by the change of variable Eq. (3.18). For this change of integration to be valid, the propagator, and hence the functions $A\left(q^{2}\right) /\left[q^{2} A\left(q^{2}\right)^{2}+B\left(q^{2}\right)^{2}\right]$ and $B\left(q^{2}\right) /\left[q^{2} A\left(q^{2}\right)^{2}+B\left(q^{2}\right)^{2}\right]$, must be analytic over the shaded region in Fig. 1,

$$
\operatorname{Re}\left(q^{2}\right)>-m_{R}^{2}+\frac{\left(\operatorname{Im}\left(q^{2}\right)\right)^{2}}{4 m_{R}^{2}}
$$

Equivalently, the function defined by

$$
\sigma_{\mathrm{Q}}(K, \kappa)=\frac{1}{i K+\Sigma(K, \kappa)},
$$

must be analytic over the shaded region $\operatorname{Im} K<0$.

The confinement criterion that $S(p)$ should be free from timelike poles on the negative real $p^{2}$ axis translates in the heavy quark case to a requirement that $\sigma_{\mathrm{Q}}$ should be free from poles on the imaginary $K$ axis. The stronger conjecture [7], that the quark propagator should be an entire function of $p^{2}$ translates in the heavy quark formalism to a conjecture that $\sigma_{\mathrm{Q}}$ should be an entire function of $K$.

From Eq. (3.23), we have that the renormalisation condition Eq. (2.4) is equivalent to

$$
\left.S(p, \mu)\right|_{p^{2}=\mu^{2}}=\left.\frac{1+\gamma_{4}}{2} \frac{1}{i K}\right|_{K=\kappa},
$$

to zeroth order in $1 / m_{R}$. Typically we choose $\kappa$ to be on the negative imaginary $K$ axis, as the heavy quark propagator asymptotes to the bare propagator as $K \rightarrow-i \infty$, as can be seen from Fig. 1.

\section{B. Heavy quark DSE}

To illustrate the derivation of the renormalised heavy quark DSE, we choose Landau gauge $(\xi=0$ in Eq. (2.13) ) and, for the time being, work with the rainbow or bare vertex approximation 


$$
\Gamma_{\mu}(p, q)=\gamma_{\mu} .
$$

Using Dirac trace identities to project out from Eqs. (2.1) to (2.3) a pair of coupled integral equations gives

$$
\begin{gathered}
A^{\prime}\left(p^{2}, \Lambda^{2}\right)= \\
1+\frac{4 Z_{1}}{3 p^{2}} \int^{\Lambda} \frac{d^{4} q}{(2 \pi)^{4}}\left[p \cdot q+2 \frac{p \cdot(p-q) q \cdot(p-q)}{(p-q)^{2}}\right] \Delta\left[(p-q)^{2}\right] \frac{A\left(q^{2}\right)}{q^{2} A^{2}+B^{2}}, \\
B^{\prime}\left(p^{2}, \Lambda^{2}\right)=4 Z_{1} \int^{\Lambda} \frac{d^{4} q}{(2 \pi)^{4}} \Delta\left[(p-q)^{2}\right] \frac{B\left(q^{2}\right)}{q^{2} A^{2}+B^{2}} .
\end{gathered}
$$

Substituting

$$
\begin{gathered}
\frac{A\left(q^{2}\right)}{q^{2} A\left(q^{2}\right)^{2}+B\left(q^{2}\right)^{2}}=\frac{1}{2 m_{R}} \frac{1}{i K^{\prime}+\Sigma\left(K^{\prime}\right)}+O\left(\frac{1}{m_{R}^{2}}\right), \\
\frac{B\left(q^{2}\right)}{q^{2} A\left(q^{2}\right)^{2}+B\left(q^{2}\right)^{2}}=\frac{1}{2} \frac{1}{i K^{\prime}+\Sigma\left(K^{\prime}\right)}+O\left(\frac{1}{m_{R}}\right),
\end{gathered}
$$

into Eqs. (3.29) and (3.30) gives

$$
\begin{aligned}
m_{R}\left[A^{\prime}\left(p^{2}, \Lambda^{2}\right)-1\right]= & \frac{2 Z_{1}}{3} \int^{\Lambda} \frac{d^{4} k^{\prime}}{(2 \pi)^{4}}\left[1+2 \frac{\left(k_{4}-k_{4}^{\prime}\right)^{2}}{\left(k-k^{\prime}\right)^{2}}\right] \\
& \times \Delta\left[\left(k-k^{\prime}\right)^{2}\right] \frac{1}{i K^{\prime}+\Sigma\left(K^{\prime}, \kappa\right)}+O\left(\frac{1}{m_{R}}\right),
\end{aligned}
$$

and

$$
B^{\prime}\left(p^{2}, \Lambda^{2}\right)=2 Z_{1} \int^{\Lambda} \frac{d^{4} k^{\prime}}{(2 \pi)^{4}} \Delta\left[\left(k-k^{\prime}\right)^{2}\right] \frac{1}{i K^{\prime}+\Sigma\left(K^{\prime}, \kappa\right)}+O\left(\frac{1}{m_{R}}\right) .
$$

From Eqs. (2.6), (2.7), (3.19), (3.20) and (3.24) we have

$$
\Sigma(K, \kappa)=\left[B^{\prime}\left(p^{2}, \Lambda^{2}\right)-m_{R} A^{\prime}\left(p^{2}, \Lambda^{2}\right)\right]-\left[p^{2} \rightarrow \mu^{2}\right],
$$

which gives

$$
\Sigma(K, \kappa)=\frac{4}{3} \int^{\Lambda} \frac{d^{4} k^{\prime}}{(2 \pi)^{4}}\left[\frac{\left|\mathbf{k}-\mathbf{k}^{\prime}\right|^{2}}{\left(k-k^{\prime}\right)^{2}} \Delta\left[\left(k-k^{\prime}\right)^{2}\right] \frac{1}{i K^{\prime}+\Sigma\left(K^{\prime}, \kappa\right)}-(K \rightarrow \kappa)\right],
$$

where $K$ on the left hand side is related to $k$ under the integrand via Eq. (3.21), and a similar definition exists relating $K^{\prime}$ and $k^{\prime}$. Here we have assumed that Eqs. (2.5) and (3.33) imply $Z_{1}=1+O\left(1 / m_{R}\right)$.

As noted above, $K$ and $k_{4}$ are interchangeable to leading order, except where they occur in the denominator of the heavy fermion propagator $1 /\left[i K^{\prime}+\Sigma\left(K^{\prime}\right)\right]$ in the integrand of Eq. (3.36). This is because, for any gluon propagator $\Delta$ with a realistic asymptotic UV 
behaviour, all powers of $k^{\prime}$ must be retained in the denominator to maintain the same degree of divergence in the heavy fermion DSE as in the original equation (2.1). With this observation, and choosing $k_{\mu}=(\mathbf{0}, K)$ and the renormalisation point $(\mathbf{0}, \kappa)$, we arrive at the leading order heavy quark DSE

$$
\begin{aligned}
\Sigma(K, \kappa)= & \frac{4}{3} \int^{\Lambda} \frac{d^{4} k^{\prime}}{(2 \pi)^{4}} \frac{1}{i k_{4}^{\prime}+k^{\prime 2} /\left(2 m_{R}\right)+\Sigma\left(k_{4}^{\prime}, \kappa\right)} \\
& \times\left\{\frac{\left|\mathbf{k}^{\prime}\right|^{2} \Delta\left[\left(K-k_{4}^{\prime}\right)^{2}+\left|\mathbf{k}^{\prime}\right|^{2}\right]}{\left(K-k_{4}^{\prime}\right)^{2}+\left|\mathbf{k}^{\prime}\right|^{2}}-(K \rightarrow \kappa)\right\} .
\end{aligned}
$$

We show in the Appendix that, with the smeared FR gluon propagator Ansatz Eq. (2.17), and assuming a hierarchy of scales

$$
m_{t},|K|,|\kappa| \ll m_{R} \ll \Lambda,
$$

the integral in Eq. (3.37) is independent of the ultraviolet cutoff $\Lambda$, and the heavy quark self energy behaves like

$$
\Sigma(K, \kappa) \sim 2 i d(\kappa-K) \ln \left(\frac{m_{R}}{m_{t}}\right)
$$

as $m_{R} \rightarrow \infty$. With the more severely truncated gaussian gluon propagator Eq. (2.15), the renormalisation point $\kappa$ can be taken to $-i \infty$ and the $k^{\prime 2} /\left(2 m_{R}\right)$ term in the denominator of the integrand ignored with impunity.

\section{Choice of renormalisation point}

At the end of the day, physical quantities must be insensitive to the choice of renormalisation point $\kappa$. In this section we note that the freedom to choose the renormalisation point is equivalent to the notion of a 'residual mass' in HQET [21, 14], that is, the notion that to zeroth order in $1 / m_{R}$, physical quantities computed in HQET do not depend on the choice of $m_{R}$.

After formally carrying out the spatial momentum integration in Eq. (3.37), one obtains an equation generically of the form

$$
\Sigma(K, \kappa)=\frac{1}{\sigma_{Q}(K, \kappa)}-i K=\int_{-\infty}^{\infty} d K^{\prime}\left[T\left(K-K^{\prime}\right)-T\left(\kappa-K^{\prime}\right)\right] \sigma_{Q}\left(K^{\prime}, \kappa\right),
$$

for some kernel $T$, and with $\sigma_{Q}$ defined by $\mathrm{Eq}(3.26)$. It is possible to show from this generic form that the effect of making a change of renormalisation point $\kappa_{\text {old }} \rightarrow \kappa_{\text {new }}$ is equivalent to a shift of the quark propagator solution along the imaginary $K$ axis:

$$
\sigma_{Q}\left(K, \kappa_{\text {new }}\right)=\sigma_{Q}\left(K-i \delta m, \kappa_{\text {old }}\right)
$$

where $\delta m$ is the solution to

$$
\frac{1}{i \kappa_{\text {new }}}=\sigma_{Q}\left(\kappa_{\text {new }}-i \delta m, \kappa_{\text {old }}\right)
$$


that is, the shift is that required to ensure that the new heavy quark propagator pass through the renormalisation point $\sigma_{Q}\left(\kappa_{\text {new }}, \kappa_{\text {new }}\right)=1 / i \kappa_{\text {new }}$. Referring to Fig. 11 we see that this is equivalent to a shift in the position of the origin of the $K$ plane along the $p^{2}$ axis corresponding to changing $m_{R}$ by an amount $\delta m$.

One can also demonstrate that the mass differences between any two heavy quark-light antiquark meson states calculated from the Bethe-Salpeter formalism set out in ref. [12] is independent of the renormalisation point.

\section{RESULTS}

Our main concern in this paper is to compare how the analytic structure of the heavy quark propagator solutions is affected by the approximations employed both for the quarkgluon vertex and the gluon propagator. In an earlier work [12 an attempt was made to study the heavy quark-light antiquark meson spectrum using a combination of rainbow DSE and ladder BSE. It was found that, if a simple gaussian Ansatz is used for the gluon propagator, complex conjugate poles occur in the heavy quark propagator which prevent solution of the meson BSE. Below we systematically explore the movement of the poles as the bare vertex of the rainbow approximation is replaced by a Ball-Chiu vertex, and as the gaussian gluon propagator is replaced by the more realistic Frank and Roberts propagator.

\section{A. Gaussian gluon propagator}

\section{Rainbow approximation}

As noted in the previous section, if the gluon propagator Eq. (2.15) is used, we may set the renormalisation point $\kappa=-i \infty$ and ignore the $k^{\prime 2} /\left(2 m_{R}\right)$ term in Eq. (3.37). Furthermore, there is no need to distinguish between the independent momentum variables $K$ and $k_{4}$. With these simplifications the Landau gauge, rainbow heavy quark DSE becomes

$$
\Sigma\left(k_{4}\right)=\frac{4}{3} \int \frac{d^{4} k^{\prime}}{(2 \pi)^{4}} \frac{\left|\mathbf{k}-\mathbf{k}^{\prime}\right|^{2}}{\left(k-k^{\prime}\right)^{2}} \frac{\Delta\left(\left(k-k^{\prime}\right)^{2}\right)}{i k_{4}^{\prime}+\Sigma\left(k_{4}^{\prime}\right)} .
$$

Choosing the infrared dominant gluon propagator Eq. (2.14), the DSE reduces to an algebraic equation with solution

$$
\Sigma\left(k_{4}\right)= \begin{cases}\frac{1}{2}\left(-i k_{4}+\sqrt{\frac{3 \mu^{2}}{4}-k_{4}^{2}}\right) & \text { if } \quad 0 \leq k_{4}<\frac{\sqrt{3} \mu}{2}, \\ \frac{i}{2}\left(-k_{4}+\sqrt{k_{4}^{2}-\frac{3 \mu^{2}}{4}}\right) & \text { if } \quad k_{4} \geq \frac{\sqrt{3} \mu}{2}\end{cases}
$$

or, using the definition (3.26),

$$
\sigma_{\mathrm{Q}}\left(k_{4}\right)= \begin{cases}\frac{8}{3 \mu^{2}}\left(-i k_{4}+\sqrt{\frac{3 \mu^{2}}{4}-k_{4}^{2}}\right) & \text { if } \quad 0 \leq k_{4}<\frac{\sqrt{3} \mu}{2}, \\ \frac{8 i}{3 \mu^{2}}\left(-k_{4}+\sqrt{k_{4}^{2}-\frac{3 \mu^{2}}{4}}\right) & \text { if } \quad k_{4} \geq \frac{\sqrt{3} \mu}{2}\end{cases}
$$


Alternatively, choosing the gaussian gluon propagator Eq. (2.15) and carrying out the $d^{3} \mathbf{k}$ integration, we obtain the integral equation [22]

$$
\begin{aligned}
& \Sigma\left(k_{4}\right)=\frac{\mu^{2}}{2 \alpha^{2} \sqrt{\pi}} \int_{-\infty}^{\infty} d k_{4} \frac{1}{i k_{4}^{\prime}+\Sigma\left(k_{4}^{\prime}\right)} \times \\
& \left\{\sqrt{\alpha}\left[\frac{\alpha}{2}-\left(k_{4}-k_{4}^{\prime}\right)^{2}\right] e^{-\left(k_{4}-k_{4}^{\prime}\right)^{2} / \alpha}+\sqrt{\pi}\left|k_{4}-k_{4}^{\prime}\right|^{3} \operatorname{erfc}\left(\frac{\left|k_{4}-k_{4}^{\prime}\right|}{\sqrt{\alpha}}\right)\right\},
\end{aligned}
$$

where $\operatorname{erfc} z=1-\operatorname{erf} z$ is the complementary error function. This equation can be solved numerically.

\section{Ball-Chiu vertex}

If any of the minimal Ball-Chiu Ansatz Eq. (2.9), the Curtis-Pennington Ansatz Eqs.(2.8) and (2.10), or the Haeri Ansatz Eq. (2.12) is used in place of the bare vertex, together with the Landau gauge gluon propagator, we obtain in place of Eq. (4.43) the equation

$$
\Sigma\left(k_{4}\right)=\frac{4}{3} \int \frac{d^{4} k^{\prime}}{(2 \pi)^{4}} \frac{\left|\mathbf{k}-\mathbf{k}^{\prime}\right|^{2}}{\left(k-k^{\prime}\right)^{2}} \frac{\Delta\left(\left(k-k^{\prime}\right)^{2}\right)}{i k_{4}^{\prime}+\Sigma\left(k_{4}^{\prime}\right)}\left[1+\frac{\Sigma\left(k_{4}\right)-\Sigma\left(k_{4}^{\prime}\right)}{i\left(k_{4}-k_{4}^{\prime}\right)}\right] .
$$

It is interesting to note that, within the set of vertex Ansätze we have considered, the heavy quark propagator is insensitive to the transverse part of the vertex. This is not difficult to understand for the Curtis-Pennington vertex, in which the transverse part is heavily damped by the presence of the factor $M^{4} \sim m^{4}$ in the denominator $d(p, q)$. However, in the case of the Haeri vertex there is no such obvious mechanism, and one is led to question whether the heavy quark propagator may be insensitive to a broad class of Ansätze satisfying the criteria specified above Eq. (2.8).

Taking the gluon propagator to be the infrared dominant form Eq. (2.14), gives the differential equation

$$
\Sigma\left(k_{4}\right)=\frac{3 \mu^{2}}{16 i} \frac{d}{d k_{4}} \ln \left[i k_{4}+\Sigma\left(k_{4}\right)\right]
$$

which, together with the boundary condition $\sigma_{\mathrm{Q}}\left(k_{4}\right) \rightarrow 0$ as $k_{4} \rightarrow-i \infty$, admits the solution

$$
\sigma_{\mathrm{Q}}\left(k_{4}\right)=\beta\left[\sqrt{\pi} e^{-\beta^{2} k_{4}^{2}}+2 i F\left(-\beta k_{4}\right)\right],
$$

where $\sigma_{Q}$ is defined by Eq. (3.26), $\beta=2 \sqrt{2} / \mu \sqrt{3}$ and

$$
F(z)=e^{-z^{2}} \int_{0}^{z} e^{t^{2}} d t=\frac{i \sqrt{\pi}}{2} e^{-z^{2}} \operatorname{erf}(-i z)
$$

is Dawson's integral. We note that this solution is an entire function of $k_{4}$, which, as pointed out earlier, is a desirable feature of a quark propagator. This comes as no surprise, as it simply the heavy quark limit of the model considered in ref. [7], in which it was demonstrated that the combination of Ball-Chiu vertex and infrared dominant gluon propagator leads to an entire function propagator for all values of the bare current quark mass. 
It is of interest to determine to what extent this analytic structure is a feature of the BallChiu vertex, and to what extent it is a feature of the infrared dominant gluon propagator. If the infrared dominant propagator is replaced by the gaussian smeared form Eq. (2.15), we obtain the integral equation

$$
\begin{aligned}
& \Sigma\left(k_{4}\right)=\frac{\mu^{2}}{2 \alpha^{2} \sqrt{\pi}} \int_{-\infty}^{\infty} d k_{4} \frac{1}{i k_{4}^{\prime}+\Sigma\left(k_{4}^{\prime}\right)} \times \\
& \left\{\sqrt{\alpha}\left[\frac{\alpha}{2}-\left(k_{4}-k_{4}^{\prime}\right)^{2}\right] e^{-\left(k_{4}-k_{4}^{\prime}\right)^{2} / \alpha}+\sqrt{\pi}\left|k_{4}-k_{4}^{\prime}\right|^{3} \operatorname{erfc}\left(\frac{\left|k_{4}-k_{4}^{\prime}\right|}{\sqrt{\alpha}}\right)\right\} \\
& \times\left[1+\frac{\Sigma\left(k_{4}\right)-\Sigma\left(k_{4}^{\prime}\right)}{i\left(k_{4}-k_{4}^{\prime}\right)}\right],
\end{aligned}
$$

which can be solved numerically.

\section{Numerical results: gaussian gluon propagator}

For the purpose of determining the analytic structure of the heavy fermion propagator obtained from the DSE with the gaussian gluon propagator Eq. (2.15), it is sufficient to look at the one parameter family of models obtained by scaling either $\mu$ or $\alpha$ to unity. We choose to scale $\mu$ to unity, which amounts to working with a set of dimensionless quantities

$$
\hat{k}_{4}=k_{4} / \mu, \quad \hat{\alpha}=\alpha / \mu^{2}, \quad \hat{\sigma}_{\mathrm{Q}}=\mu \sigma_{\mathrm{Q}}
$$

This choice enables us to recover the infrared dominant model in the limit $\alpha \rightarrow 0$.

In Figs. 2 and 3 we plot the heavy quark self energy $\Sigma\left(k_{4}\right)$ as a function of real $k_{4}$ obtained from the bare vertex DSE Eq. (4.46) and the Ball-Chiu vertex DSE Eq. (4.51) for $\hat{\alpha}=1,2$ and 3 . These results are obtained by iterating from an initial guess and using a Simpson's rule quadrature. We find that the derivative-like terms in Eq. (4.51) prevent a numerical solution for values of $\hat{\alpha}$ less than 1, as numerical noise in the function values becomes unstable with respect to iteration at small values of $k_{4}$. This problem is a general feature of numerical treatments of DSEs with Ball-Chiu-like vertices. Also plotted are the $\hat{\alpha}=0$ analytic results Eqs. (4.44) and (4.49). In all cases the self energy is characterised by a real part which peaks at zero and an imaginary part which peaks near the typical scale of the model $k_{4} \sim \mu$. The self energy for negative real $k_{4}$ can be obtained from these results using the reflection property $\Sigma\left(-k_{4}^{*}\right)=\Sigma\left(k_{4}\right)^{*}$.

To solve for the heavy quark propagator away from the real $k_{4}$ axis we shift the contour of integration into the complex plane parallel to the real $k_{4}$ axis and again solve iteratively. We note that, to determine $\Sigma\left(k_{4}\right)$ for complex arguments, it is necessary to move the contour of integration to pass through the point $k_{4}$. This is because the radial part of the $d^{3} \mathbf{k}^{\prime}$ integration, carried out in going from Eq. (4.43) to Eq. (4.46) or from Eq. (4.47) to Eq. (4.51), creates a pinch singularity at $k_{4}^{\prime}=k_{4}$ in the error function term in Eq. (4.46) or Eq. (4.51).

We have carried out a search for poles in the propagator function $\sigma_{Q}\left(k_{4}\right)$ for a range of values of $\alpha$ for both the bare and Ball-Chiu vertex. Our results are listed in Table $\mathbb{1}$. In all cases we find that the only observed poles occur for $\operatorname{Im} k_{4}>0$, and that $\sigma_{Q}$ dies away to small values and is free from singularities over that part of the shaded region in 
Fig. 1 accessible to our computer program. Of course we are unable to pass the contour of numerical integration through the pole itself, and these results are attained by extrapolation from results of contours which we gradually moved deeper into the complex plane. We were unable to obtain a reasonable extrapolation for the Ball-Chiu vertex at $\alpha=1$, again because of the iterative instability problem associated with the derivative-like term in Eq. (4.51).

We also list in Table [ the results of using the model gluon propagator

$$
g^{2} D_{\mu \nu}\left(k^{2}\right)=\delta_{\mu \nu} \Delta_{\mathrm{G}}\left(k^{2}\right),
$$

where $\Delta_{\mathrm{G}}$ is given by Eq. (2.15). Forms such as Eq. (4.53) are frequently used in phenomenological modelling (see for instance ref. [2]) and are sometimes referred to as propagators in a 'Feynman-like gauge', though of course they are generally not of the form of Eq. (2.13). The sole advantage of the Feynman-like gauge is that it leads to considerably simplified calculations. In our case it is possible to locate poles more accurately because there is no pinch singularity requiring the contour of integration to pass through the point in question. Once the propagator has been solved on the real $k_{4}$ axis, the value of the propagator can be calculated at any point in the complex plane by integrating once along the real axis. Nevertheless, we have also repeated our pole calculations by shifting the contour and extrapolating as in the Landau gauge case as a check on the the consistency of the two methods and find that they agree to within the accuracy given in Table 1 of the corresponding Landau gauge results. A Feynman-like gauge propagator was also used in ref. [12] dealing with the ladder Bethe-Salpeter equation for the heavy quark-light antiquark system. There it was demonstrated that the model with bare quark gluon vertex and gaussian Feynman-like gauge gluon propagator had no solutions because of poles in the heavy and light quark propagators. Ideally, one would like improvements in the DSE approximations to move the poles further from the real $k_{4}$ axis to avoid the region of the $k_{4}$ plane sampled by a Bethe-Salpeter calculation.

From Table \we conclude that simply replacing the bare vertex by the Ball-Chiu vertex in itself does nothing to improve the pole structure of the heavy quark propagator, either for the Landau gauge gluon propagator or the Feynman-like gluon propagator. In particular we find that, as the gaussian width $\alpha$ increases, a mass pole pole moves in along the imaginary $k_{4}$ axis. A pole on the imaginary axis indicates that the fermion can propagate as a free particle, and the position on the positive imaginary axis gives the contribution to the quark mass from the dynamical self dressing. For the bare vertex, the pole splits into conjugate pairs either side of the imaginary axis as $\alpha$ decreases. In this instance the quark becomes a confined particle. Numerical difficulties described above prevented us from confirming that the same situation occurs in the case of the Ball-Chiu vertex. As $\alpha \rightarrow 0$ we must recover the solution Eq. (4.49), which is an entire function with an essential singularity at infinity.

We see from Table 1 that, when poles occur, their position remains almost unchanged in going from Landau to Feynman-like gauge if the Ball-Chiu vertex is used, but not if the bare vertex is used. In a properly formulated gauge covariant calculation, the position of any propagator mass pole should be independent of the gauge fixing procedure [23]. While we certainly do not claim that that our treatment is gauge covariant, it is amusing to note that replacing the bare vertex by the Ball-Chiu vertex (and Feynman gauge by the computationally convenient Feynman-like gauge) appears to go some way towards satisfying this requirement. 


\section{B. Frank and Roberts gluon propagator}

We now return to the renormalised DSE Eq. (3.37) with the gluon propagator $\Delta$ set equal to the smeared FR Ansatz Eq. (2.17). For numerical simplicity we shall restrict ourselves to the rainbow approximation $\Gamma_{\mu}(p, q)=\gamma_{\mu}$. We set

$$
K=X+i Y, \quad \kappa=i \eta .
$$

Assuming the contour of integration can be deformed to pass through $k_{4}^{\prime}=K$ for the integral of the first term in chain brackets in Eq.(3.37), and through $k_{4}^{\prime}=\kappa$ for the second term, we further set

$$
k_{4}^{\prime}=x+i Y \quad \text { and } \quad k_{4}^{\prime}=x+i \eta
$$

respectively in each of these two terms. We also make the replacement $k^{\prime 2} /\left(2 m_{R}\right) \rightarrow\left(x^{2}+\right.$ $\left.\left|\mathbf{k}^{\prime}\right|^{2}\right) /\left(2 m_{R}\right)$ without affecting $\Sigma$ to leading order. This gives

$$
\begin{aligned}
& \Sigma(X+i Y, i \eta)=\frac{4}{3} \int_{-\infty}^{\infty} \frac{d x}{2 \pi} \int \frac{d^{3} \mathbf{k}^{\prime}}{(2 \pi)^{3}} \\
& \left\{\frac{1}{i x-Y+\left(x^{2}+\left|\mathbf{k}^{\prime}\right|^{2}\right) /\left(2 m_{R}\right)+\Sigma(x+i Y)} \frac{\left|\mathbf{k}^{\prime}\right|^{2}}{(x-X)^{2}+\left|\mathbf{k}^{\prime}\right|^{2}} \Delta\left[(x-X)^{2}+\left|\mathbf{k}^{\prime}\right|^{2}\right]\right. \\
& \left.-\frac{1}{i x-\eta+\left(x^{2}+\left|\mathbf{k}^{\prime}\right|^{2}\right) /\left(2 m_{R}\right)+\Sigma(x+i \eta)} \frac{\left|\mathbf{k}^{\prime}\right|^{2}}{x^{2}+\left|\mathbf{k}^{\prime}\right|^{2}} \Delta\left[x^{2}+\left|\mathbf{k}^{\prime}\right|^{2}\right]\right\} .
\end{aligned}
$$

For the purpose of carrying out the numerics, it is convenient to change to the polar coordinates $x=r \cos \phi,\left|\mathbf{k}^{\prime}\right|=r \sin \phi$, giving finally

$$
\begin{aligned}
& \Sigma(X+i Y, i \eta)=\frac{1}{3 \pi^{3}} \int_{0}^{\infty} d r \int_{0}^{\pi} d \phi r^{3} \sin ^{2} \phi \\
& \left\{\frac{1}{i r \cos \phi-Y+r^{2} /\left(2 m_{R}\right)+\Sigma(r \cos \phi+i Y)} \frac{r^{2} \sin ^{2} \phi}{r^{2}-2 X r \cos \phi+X^{2}} \Delta\left(r^{2}-2 X r \cos \phi+X^{2}\right)\right. \\
& \left.-\frac{1}{i r \cos \phi-\eta+r^{2} /\left(2 m_{R}\right)+\Sigma(r \cos \phi+i \eta)} \sin ^{2} \phi \Delta\left(r^{2}\right)\right\} .
\end{aligned}
$$

This equation is first solved numerically along the line $\operatorname{Im} K=\eta$, (i.e. $Y=\eta$ ), and the function $\Sigma$ along this line is stored for subsequent calculations at arbitrary $Y$.

\section{Numerical results: Frank and Roberts propagator}

We have numerically solved Eq. (4.57) over a region of the complex $K$ plane with the smeared FR gluon propagator $\Delta_{\mathrm{GFR}}$. Our parameter choices are $m_{t}=0.69 \mathrm{GeV}$, in agree-

ment with ref. [16], and $\alpha=16 m_{t}^{2} \hat{\alpha} d / 3=0.5643(\mathrm{GeV})^{2}$ corresponding to $\hat{\alpha}=0.5$. The choice of $\alpha$ is designed so that a comparison can be made between the full FR propagator and the gaussian propagator Eq. (2.15) obtained by keeping only the first term in Eq. (2.17). From Table [ w we know that gaussian propagator $\Delta_{\mathrm{G}}$ with the parameter choice $\hat{\alpha}=0.5$ results in a pair of conjugate poles in the heavy quark propagator. The heavy quark mass is set to $m_{R}=5.0 \mathrm{GeV}$. 
In Fig. 4 we plot the modulus $\left|\sigma_{Q}(K)\right|$ of the heavy quark propagator for the full gaussian FR propagator $\Delta_{\text {GFR }}$ and in Fig. 5 plot the same quantity using only the gaussian part of $\Delta_{\mathrm{G}}$ with the parameters otherwise unchanged. Both calculations have been done using the renormalisation point $\kappa=-1.0 i \mathrm{GeV}$, and to clarify the comparison the same region of the $K$ plane is displayed in both plots. The calculation of $\sigma_{Q}(K)$ involves a shift of integration path to a contour parallel to the real axis passing through the point $K$. Deforming the contour to include points behind the pole is a numerically tedious exercise which is unlikely to enhance our understanding, so no results are given for the part of the $K$ plane in Fig. 5 .

In the process of carrying out our computations, we have observed that the shift property resulting from changes of renormalisation point, namely Eq. (3.41), is indeed respected by our numerical solutions. In fact, the full plot in Fig. 1 was pieced together by altering the renormalisation point to obtain solutions in strips of the complex plane parallel to the real axis, and using Eq. (3.42) to match solutions where strips overlapped.

We note a clear movement of the propagator pole further away from the real $K$ axis when the asymptotic ultraviolet tail is included in the gluon propagator. Since it is the proximity of the pole to the real axis which prevented a solution to BSE in our earlier studies, this suggests that this movement of the pole portends well for future possible studies of heavy quark mesons if careful attention is paid to the asymptotic ultraviolet behaviour of the gluon propagator.

However, a note of caution is in order. A different choice of renormalisation point would result in the plots in Figs. 1 and 5 shifting by different amounts respectively along the imaginary axis, ensuring that both plots pass through the same point $\sigma_{Q}(\kappa)=1 / i \kappa$. Consequently, the actual amount by which the pole moves away from the real $K$ axis as a result of adding an asymptotic tail to the gluon propagator is an artefact of the choice of renormalisation point, though the movement will always be away from the real axis. Of course only by carrying though the BSE calculation completely can one say for certain whether bound state meson solutions can be obtained.

\section{CONCLUSIONS AND OUTLOOK}

We have explored the analytic structure of heavy quark propagators following a recently proposed formalism which borrows ideas both from the DSE technique and HQET. It is our belief that, if the successes of HQET are to be properly understood, we must first understand how the non-perturbative dynamics of QCD affect the heavy quark propagator. Within the light quark sector the analytic structure of the quark propagator is perhaps best understood in terms of model Dyson-Schwinger equations. It is therefore a worthwhile exercise to extend the DSE technique to the heavy quark limit.

The initial attempt in this direction [12] failed essentially because the approximations used led to spurious propagator poles which prevented solution of the bound state BetheSalpeter equations. Two approximations were involved: modelling of the quark-gluon vertex and of the the gluon propagator. We have focused on each of these aspects in turn in this paper. In order to deal with an improved Ansatz for the gluon propagator with a realistic asymptotic ultraviolet behaviour, it has been necessary to formulate a properly renormalised version of the heavy quark DSE technique proposed in ref. [12]. As an interesting corollary to 
our formalism we observe that the freedom to choose the renormalisation point is tantamount to the freedom in zeroth order HQET to choose the heavy quark mass up to a residual mass.

We have first examined the effect of replacing the bare vertex with Ansätze based on the Ball-Chiu vertex [15], which is primarily designed to satisfy the Ward-Takahashi identity. Specifically, we have considered the minimal Ball-Chiu vertex and two variants: that proposed by Curtis and Pennington [17] and that proposed by Haeri [18]. The two variants differ from the minimal vertex by the inclusion of extra transverse components. We find that, to zeroth order in the inverse of the heavy quark mass, the heavy quark propagator is insensitive to which of the three above Ansätze is used. One is led to question to what extent transverse additions to the minimal Ball-Chiu vertex can be ignored in determining the leading order heavy quark propagator. If they can be be ignored in general there are immediate benefits in using the heavy fermion limit as a test-case for studies of confining field theories.

In our numerical calculations we began with the Landau gauge form of the model gaussian gluon propagator $\Delta_{G}$, Eq. (2.15), which was employed (together with the bare vertex) in previous studies [12]. Unfortunately, we find no improvement in the propagator pole structure in going from the bare vertex to the Ball-Chiu vertex while maintaining a gaussian gluon propagator. That is to say, timelike mass poles indicating non-confinement, or conjugate poles which are likely to interfere with the successful solution to bound state problems, are not removed simply by improving the quark gluon vertex Ansatz alone. However, in the limit in which the width of the gaussian gluon propagator is taken to zero (the 'infrared dominant model'), we do obtain an entire function heavy quark propagator, free from singularities except an essential singularity at infinity. This is consistent with the equivalent finite quark mass calculation [7], and may provide a useful propagator for phenomenological modelling purposes.

We conclude then that it is most likely the remaining approximation, namely the gaussian model gluon propagator, which is responsible for the poor analytic structure previously obtained for the quark propagator. To explore this possibility, we have replaced the simple gaussian gluon propagator Ansatz of ref. [12] by a gaussian smeared version of the more sophisticated Frank and Roberts Ansatz $\Delta_{\mathrm{FR}}$ given by Eq. (2.16). In this case, convergence of the integral in the DSE which is lost by naively retaining only the lowest order of the $1 / m_{R}$ expansion of the quark propagator must be restored by judiciously including at least the spatially dependent $O\left(1 / m_{R}\right)$ part:

$$
S(p)=\frac{1+\gamma_{4}}{2} \frac{1}{i k_{4}+|\mathbf{k}|^{2} / 2 m_{R}+\Sigma\left(k_{4}\right)}+O\left(m_{R}^{-1}\right) .
$$

The renormalised current quark mass $m_{R}$ then becomes an ultraviolet regulator, and in the case of the FR propagator the lowest order contribution to the mass expansion of the heavy quark self energy behaves as $\ln \left(m_{R} / m_{t}\right)$, where $m_{t}$ delineates the scale at which the asymptotic ultraviolet behaviour of the FR propagator sets in.

Our numerical solutions of the heavy quark DSE show a clear movement of the offensive propagator poles away from that part of the complex momentum plane likely to be sampled by a Bethe-Salpeter calculation of heavy quark meson states. However we caution that the amount by which the poles shift is, strictly speaking, dependent on the choice of renormalisation point. Without carrying through the the Bethe-Salpeter analysis one cannot say for 
sure that the problem is solved. A further study of the BSE for heavy mesons is expected to be the focus of future work.

Our work has also thrown up a couple of other interesting questions worthy of attention. Firstly, it should be possible to check directly to what extent the propagator pole structure is invariant with respect to the choice of gauge fixing parameter $\xi$. As the postions of propagator poles should be gauge independent [23], this provides a straightforward measure of the ability of a particular vertex Ansatz to respect gauge covariance of the model.

Secondly, one is led to question the meaning of propagator poles obtained from a Euclidean DSE formalism. Whether propagator poles obtained in this way are an artefact of the approximations used or whether they are a genuine property of quark propagators has been an open question for some time [1,10 $\|^{2}$. It is possible that the heavy fermion limit may help to shed some light on this problem by developing a Bethe-Salpeter formalism for heavy quarkonium states. It is well known that the non-relativistic limit of the Bethe-Salpeter equation for a heavy fermion-heavy antifermion bound state can be written in the form of a Schrödinger equation [25]. The derivation typically assumes physical mass poles in the fermion propagators whose residues contribute to the resulting Schrödinger equation. An analogous derivation for the case of propagator poles which have moved off the timelike momentum axis as a result of a confining gluon propagator may help both with interpretation of quark propagator poles and with understanding the success of heavy quark potential models.

\section{ACKNOWLEDGEMENT}

The author is grateful to C. D. Roberts and P. Maris for helpful discussions.

\section{APPENDIX: CONVERGENCE OF THE HEAVY QUARK DSE}

Consider the heavy quark DSE Eq. (3.37), and suppose we assume for the gluon propagator Ansatz the asymptotic ultraviolet behaviour

$$
\Delta\left(k^{2}\right) \sim \frac{4 \pi^{2} d}{k^{2}} \quad \text { for } k^{2}>m_{t}^{2},
$$

where $m_{t}$ is a scale parameter typically of the order of $1 \mathrm{GeV}$. This is the behaviour exhibited by the smeared FR Ansatz Eq. (2.17). We demonstrate here that the right hand side of Eq. (3.37) is the finite difference of two logarithmically divergent integrals.

We begin with the change of variables

$$
k_{4}^{\prime}=r \cos \phi, \quad\left|\mathbf{k}^{\prime}\right|=r \sin \phi,
$$

\footnotetext{
${ }^{2}$ In an interesting recent development, McKay and Munczek [24 have examined the analytic structure of quark propagators when an extra constraint that solutions of the DSE should be Fourier transformable is imposed.
} 
and hence

$$
\int^{\Lambda} d^{4} k^{\prime}=4 \pi \int d k_{4}^{\prime} \int d\left|\mathbf{k}^{\prime}\right|\left|\mathbf{k}^{\prime}\right|^{2}=4 \pi \int_{0}^{\Lambda} d r \int_{0}^{\pi} d \phi r^{3} \sin ^{2} \phi
$$

This gives

$$
\begin{aligned}
\Sigma(K, \kappa)= & \frac{1}{3 \pi^{3}} \int_{0}^{\Lambda} d r \int_{0}^{\pi} d \phi \frac{r^{5} \sin ^{4} \phi}{i r \cos \phi+r^{2} /\left(2 m_{R}\right)+\Sigma(r \cos \phi)} \\
& \times\left[\frac{\Delta\left(K^{2}-2 K r \cos \phi+r^{2}\right)}{K^{2}-2 K r \cos \phi+r^{2}}-(K \rightarrow \kappa)\right] .
\end{aligned}
$$

Implicit in this equation is a hierarchy of scales given by Eq. (3.38). Simply counting powers of $r$ in the integrand, we see that each of the two terms diverges as

$$
m_{R} \int^{\Lambda} \frac{d r}{r} \sim m_{R} \ln \Lambda
$$

On the other hand consider the difference of the two terms. For $r>m_{t}$ the part in square brackets is

$$
\begin{aligned}
& {\left[\frac{4 \pi^{2} d}{\left(K^{2}-2 K r \cos \phi+r^{2}\right)^{2}}-(K \rightarrow \kappa)\right]} \\
& =4 \pi^{2} d\left[\frac{4(K-\kappa) \cos \phi}{r^{5}}+\frac{2\left(K^{2}-\kappa^{2}\right)\left(6 \cos ^{2} \phi-1\right)}{r^{6}}\right]+O\left(\frac{1}{r^{7}}\right) .
\end{aligned}
$$

Neglecting $\Sigma(r \cos \phi)$ in Eq. (A.4) for large $r$, we can approximate the propagator contribution to the integrand by

$$
\frac{1}{i r \cos \phi+r^{2} /\left(2 m_{R}\right)}=-\frac{i \cos \phi-r / 2 m_{R}}{r\left(\cos ^{2} \phi+r^{2} / 4 m_{R}^{2}\right)} .
$$

Then, taking into account the hierarchy (3.38), the contribution to the integrand for $r>m_{t}$ is approximately

$$
\frac{16 i d(\kappa-K)}{3 \pi} \int_{m_{t}}^{\infty} \frac{d r}{r} \int_{0}^{\pi} d \phi \frac{\sin ^{4} \phi \cos ^{2} \phi}{\cos ^{2} \phi+r^{2} / 4 m_{R}^{2}}
$$

giving

$$
\Sigma(K, \kappa) \sim 2 i d(\kappa-K) \ln \left(\frac{m_{R}}{m_{t}}\right) \quad \text { as } m_{R} \rightarrow \infty .
$$

The last step can be achieved using the crude approximation

$$
\frac{\cos ^{2} \phi}{\cos ^{2} \phi+r^{2} / 4 m_{R}^{2}} \approx \begin{cases}1 & \text { if } r<4 m_{R} \\ \left(4 m_{R}^{2} / r^{2}\right) \cos ^{2} \phi & \text { if } r>4 m_{R}\end{cases}
$$




\section{REFERENCES}

[1] C. D. Roberts and A. G. Williams, Prog. Part. and Nucl. Phys. 33, 475 (1994).

[2] C. J. Burden, et al., Phys. Rev. C 55, 2649 (1997); R. T. Cahill and S. M. Gunner, Phys. Lett. B 359, 281 (1995).

[3] P. Jain and H. J. Munczek, Phys. Rev. D 48, 5403 (1993), and references therein, P. Maris and C. D. Roberts, $\pi$ - and K-meson Bethe-Salpeter amplitudes, nucl-th/9708029.

[4] C. D. Roberts, Nucl. Phys. A 605, 475 (1996); C. J. Burden, et al., Phys. Lett. B 371, 163 (1996).

[5] C. D. Roberts, A. G. Williams and G. Krein, Int. J. Mod. Phys. A 7, 5607 (1992).

[6] H. Munczek, Phys. Lett. B 175, 215 (1986).

[7] C. J. Burden, C. D. Roberts and A. G. Williams, Phys. Lett. B 285, 347 (1992).

[8] P. Maris and H. A. Holties, Int. J. Mod. Phys. A 7, 5369 (1992).

[9] S. J. Stainsby, and. R. T. Cahill, Int. J. Mod. Phys. A 77541 (1992).

[10] P. Maris, Phys. Rev. D 504189 (1994), and references therein.

[11] T. W. Allen, and. C. J. Burden, Phys. Rev. D 535842 (1996).

[12] C. J. Burden and D.-S. Liu, Phys. Rev. D 55, 367 (1997).

[13] R. Delbourgo and D.-S. Liu, Phys. Rev. D 56, 27 (1997).

[14] M. Neubert, Phys. Rep. 245, 259 (1994).

[15] J. S. Ball and T.-W. Chiu, Phys. Rev. D 22, 2542 (1980).

[16] M. R. Frank and C. D. Roberts, Phys. Rev. C 53, 390 (1996).

[17] D. C. Curtis and M. R. Pennington, Phys. Rev. D 42, 4165 (1990); Phys. Rev. D 46, 2663 (1992).

[18] B. Haeri, Phys. Rev. D 43, 2701 (1991).

[19] H. Munczek (private communication). For a summary of the proof of equivalence between the Haeri vertex and the spectral representation, see C. J. Burden and C. D. Roberts, Phys. Rev. D 47, 5581 (1993).

[20] H. J. Munczek and A. M. Nemirovsky, Phys. Rev. D 28, 181 (1983); R. T. Cahill and C. D. Roberts, Phys. Rev. D 32, 2419 (1985).

[21] A. F. Falk, M. Neubert and M. Luke, Nucl. Phys. B 388, 363 (1992).

[22] I. S. Gradshteyn and I. M. Ryzhik, Table of integrals, series and products, Academic, New York, 1980, Eq. (3.446.2).

[23] D. Atkinson and M. P. Fry, Nucl. Phys. B 156, 301 (1979).

[24] D. McKay and H. Munczek, Phys. Rev. D 55, 2455 (1997).

[25] D. Flamm and F. Schöberl, Introduction to the quark model of elementary particles, (Gordon and Breach, New York, 1982). 


\section{TABLES}

TABLE I. Position $k_{4} / \mu$ of poles in the heavy quark propagator closest to the real $k_{4}$ axis, using the gaussian gluon propagator Ansatz $\Delta_{\mathrm{G}}$. Numerical instabilities prevent an accurate location of the pole in the case indicated by a question mark.

\begin{tabular}{|ccccc|}
\hline \hline & \multicolumn{2}{c}{ Landau gauge } & \multicolumn{2}{c|}{ Feynman-like gauge } \\
\hline$\hat{\alpha}=\alpha / \mu^{2}$ & bare vertex & BC vertex & bare vertex & BC vertex \\
\hline 0.5 & $\pm 0.510+0.415 i$ & $?$ & $\pm 0.5466+0.5109 i$ & $?$ \\
1 & $\pm 0.378+0.506 i$ & $?$ & $\pm 0.3383+0.6758 i$ & $0.4429 i$ \\
2 & $0.466 i$ & $0.31 i$ & $0.3844 i$ & $0.3130 i$ \\
3 & $0.307 i$ & $0.252 i$ & $0.2880 i$ & $0.2554 i$ \\
4 & $0.251 i$ & $0.219 i$ & $0.2408 i$ & $0.2210 i$ \\
\hline \hline
\end{tabular}




\section{FIGURES}

FIG. 1. The change of variables $p^{2} \rightarrow K$ used to represent the heavy quark propagator in the region of the bare fermion mass pole $p^{2}=-m_{R}^{2}$. For the change of variables in the DSE to be valid, the quark propagator must be analytic over the shaded region.

FIG. 2. The heavy quark self energy $\Sigma\left(k_{4}\right)$, from the DSE in Landau gauge with a bare quark-gluon vertex and gaussian gluon propagator with parameters: $\mu=1$ and $\alpha=0$ (solid curve), 1 (long dashes), 2 (short dashes) and 3 (dotted curve). The upper curves are Re $\Sigma$ and the lower curves $\operatorname{Im} \Sigma$.

FIG. 3. The same as Fig. 2, except with the Ball-Chiu quark-gluon vertex.

FIG. 4. The modulus $\left|\sigma_{Q}(K)\right|$ of the heavy quark propagator in the complex $K$ plane obtained by solving the heavy quark DSE in rainbow approximation with a gaussian smeared Frank and Roberts Ansatz $\Delta_{\mathrm{GFR}}$ for the gluon propagator. Input parameter values are given in the text.

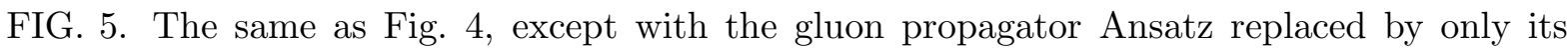
Gaussian part $\Delta_{\mathrm{G}}$, and all parameter values otherwise unchanged. 


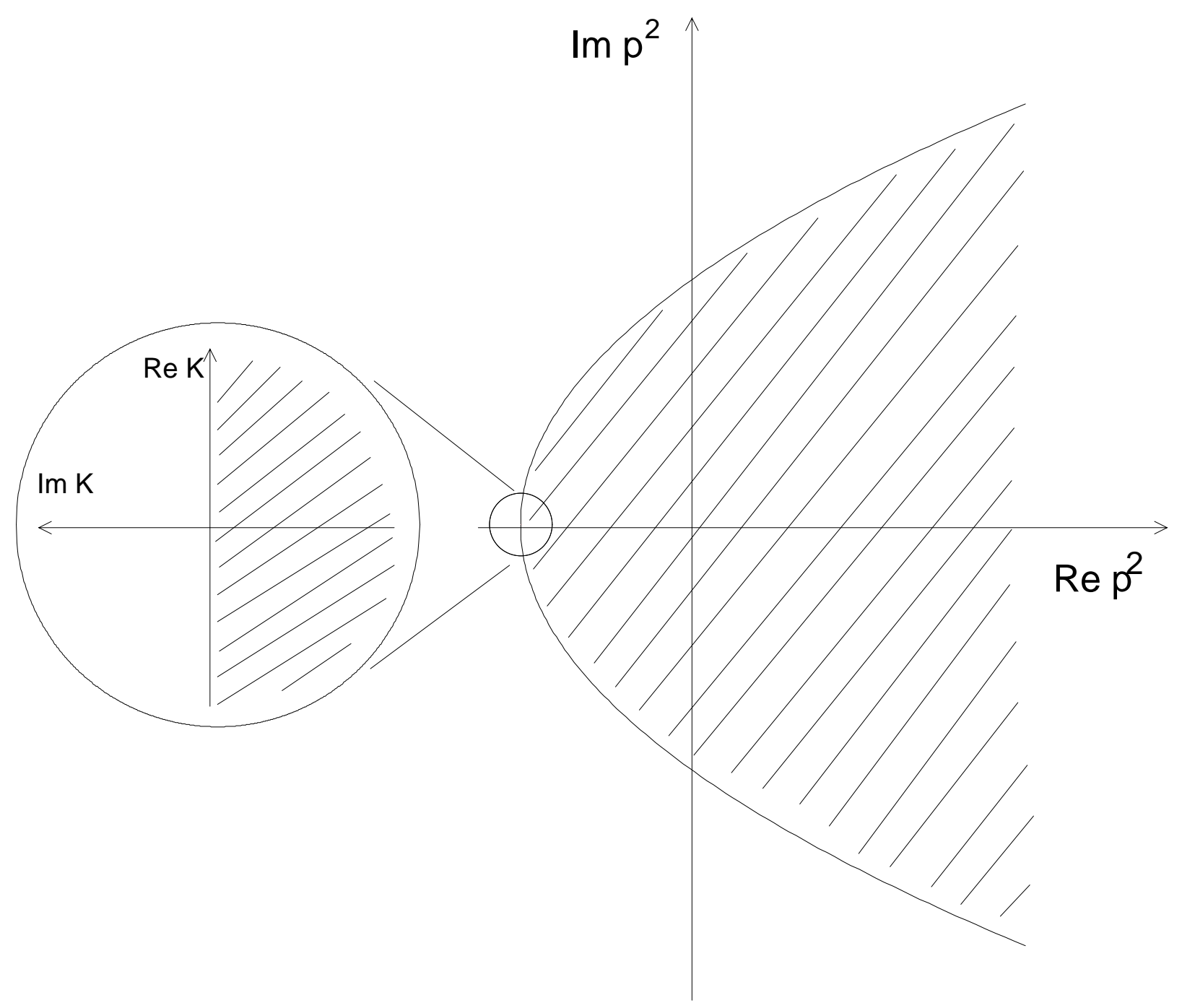




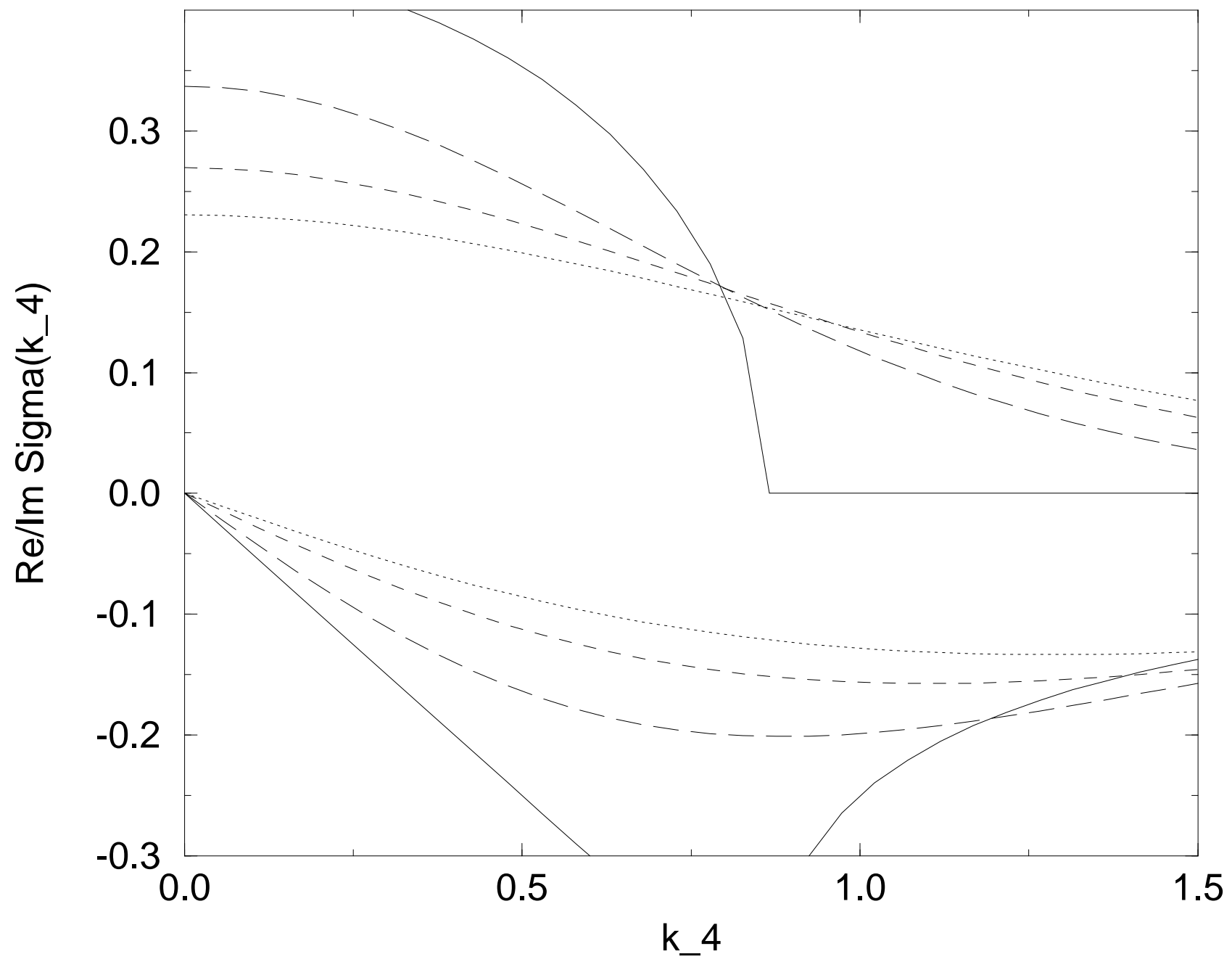




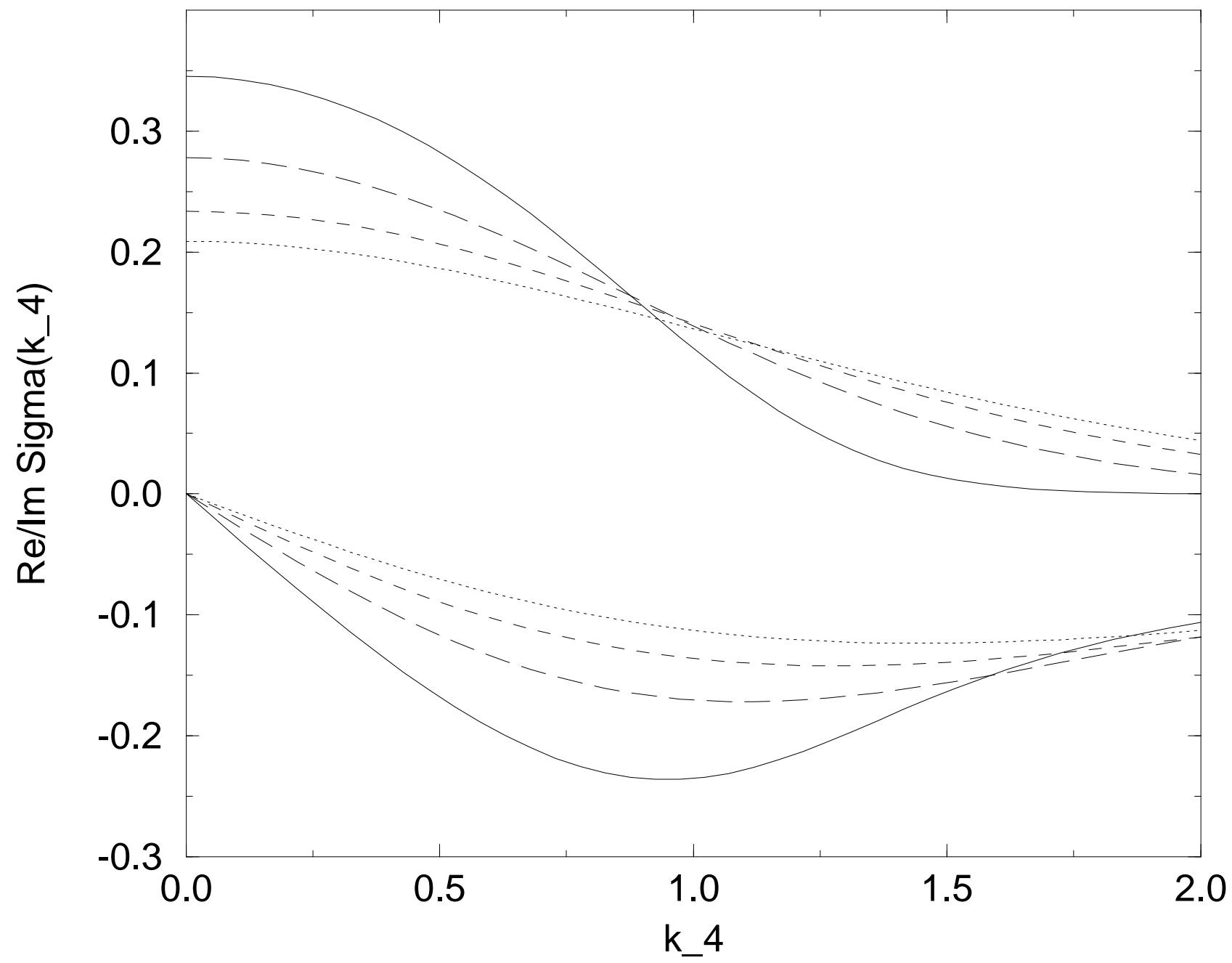




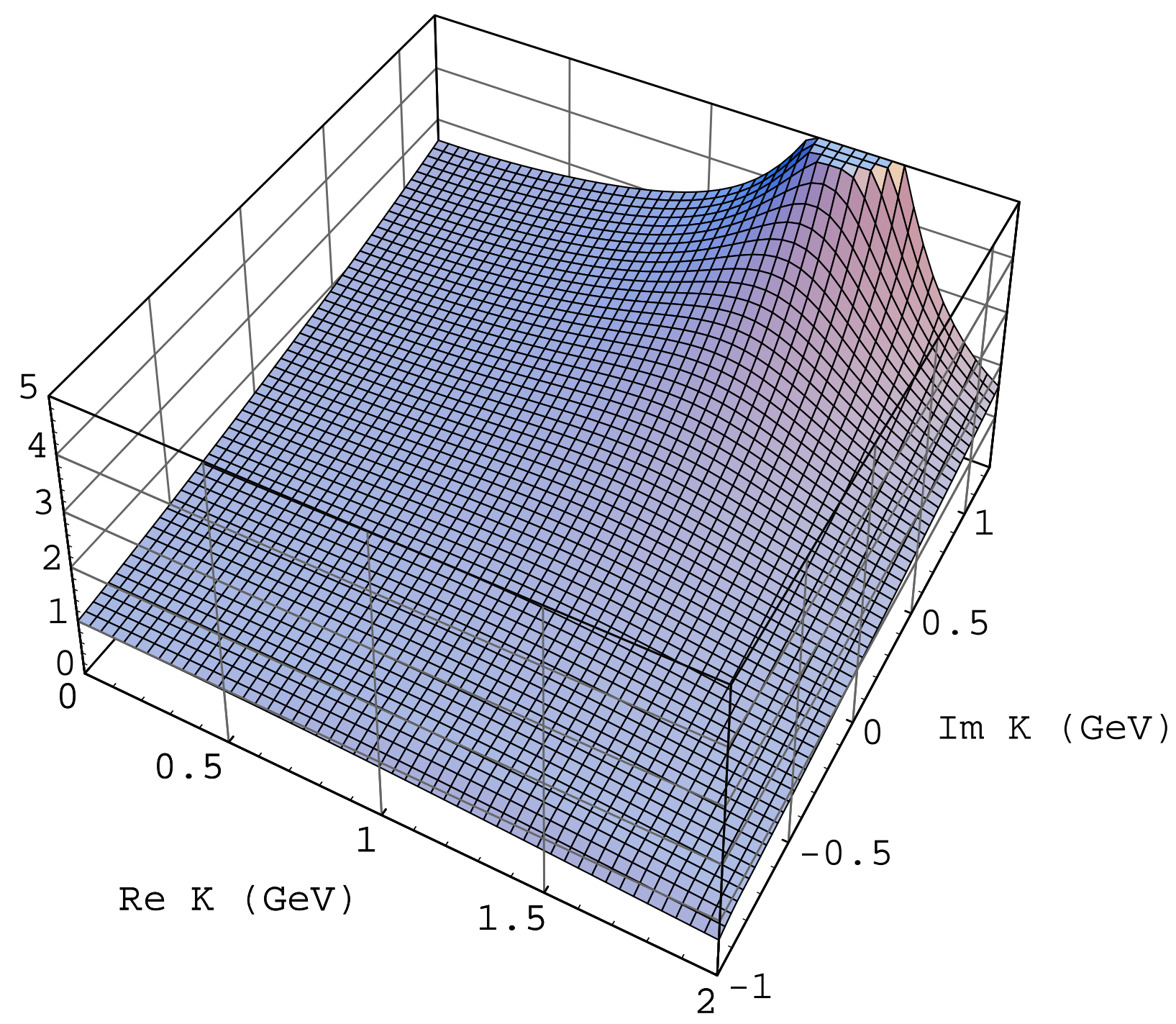




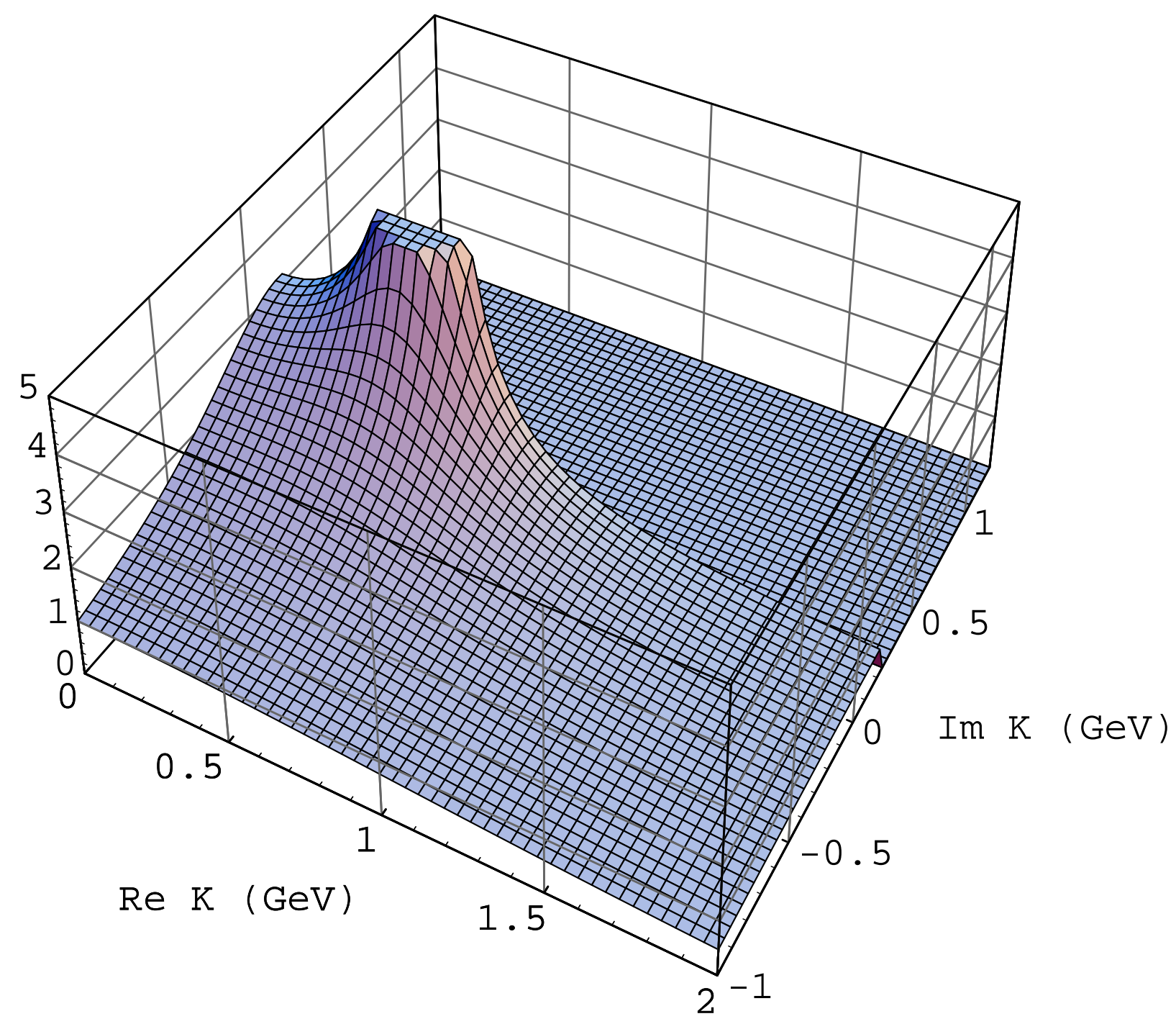

